\title{
Originals
}

\section{Statistical Property for Processing Gain of Lofargram Smoothing using Morphological Filter}

\author{
Kazuyoshi Mori \\ (National Defense Academy ) \\ (Former Maizuru National College of Technology) \\ e-mail; kmori@nda.ac.jp \\ Minoru Matsuda \\ (Osaka Electro-Communication University) \\ (Received at 5th April 2001)
}

\begin{abstract}
The methods for analyzing the temporal structures of passive sonar signals employ spectral analyses of the received signals. One of these methods is called the lofargram (low-frequency analysis record-gram). Fine-quality lofargrams are generally obtained by using averaging procedure, which constitutes a data smoothing method. However, the procedure causes the impulse noise to spread across the lofargram's time-axis. This makes it difficult to distinguish a target signal from a received signal on the lofargram. Morphological filtering is a nonlinear signal transformation that locally modifies the geometric features of signals ${ }^{4}$. Morphological opening filter can be used effectively to suppress positive impulse noise in signals and images ${ }^{5}$. The authors have proposed a method for smoothing a lofargram using morphological opening filter instead of averaging procedure. The effective improvements in processing gain and time resolution obtained in the experiment are compared with the results obtained using averaging procedure.

This paper describes some statistical properties of the processing gain of a lofargram obtained using morphological opening filter. The processing gain is defined in the case of the line structuring element, and its theoretical equation is derived when the source signal is Gaussian noise. The results of the computer simulation agree approximately with the theoretical value obtained by this equation.
\end{abstract}

Key words: Passive Sonar, Lofargram, Smoothing, Morphological Filter, opening, Processing Gain

\section{Introduction}

The temporal structure of passive sonar signals are identified by methods of spectral analysis of the received signals. The low-frequency analysis recordgram (lofargram) is one of these methods ${ }^{1), 2), 3)}$. Finequality lofargrams are usually obtained through averaging procedure, which constitutes a data smoothing method. However, this procedure spreads impulse noise across the time-axis on the lofargram, making it difficult to distinguish a target signal from a received signal on the lofargram. Morphological fil- tering is a nonlinear signal transformation that locally modifies the geometric features of signals ${ }^{4}$. Morphological opening filter can be used effectively to suppress positive impulse noise in signals and images $^{5)}$. In the present report, the authors propose a smoothing method of lofargram using morphological opening filter instead of averaging procedure. The resulting effective improvements in processing gain and time resolution are compared with the results obtained using averaging procedure ${ }^{10)}, 11$.

In averaging procedure, as the analyzing width increases, 
only the variance of the noise component is reduced, with the noise component nearing the average level. On the other hand, when morphological opening filter is used, both the average and the variance are reduced, and the noise suppression characteristics are more effective than those associated with averaging procedure. However, these characteristics were obtained as the results of numerical experiments using computer simulation, so their theoretical confirmations are necessary. Therefore, the statistical characteristics of morphological filtering must be clarified. Several researchers have made efforts to solve this problem and have achieved some useful results. Stevenson and Arce studied several statistical properties of 1-D multilevel close-opening and open-closing operations ${ }^{6}$. Yli-Harja et al. have shed some light on the statistical properties of basic morphological operation by utilizing the general expression for the output distribution of stack filter ${ }^{7}$. Wang et al. have clarified the statistical properties of binary and multilevel basic morphological operations, and reported the results of several typical probability density functions, including Gaussian ${ }^{8}$.

In this paper, some statistical properties of the processing gain of a lofargram using morphological opening filter are described. First, the probability density function of the auto-spectrum is transformed into that of the lofargram output using the statistical properties of opening filter at the line structuring element derived by Wang et al. We then describe the process to derive the theoretical equations of the probability density function of the lofargram output, including the average, the variance, and the processing gain. Next, assuming the auto-spectrum is a random variable that obeys $\chi^{2}$ distribution when the source signal is Gaussian noise, the theoretical equation of the processing gain is derived. Finally, the results of the computer simulation are reported, revealing that the numerical calculation value agrees approximately with the theoretical value obtained by this equation.

\section{Processing gain of a lofargram using morphological opening filter}

Fig. 1 shows two block diagrams of a lofargram, one smoothed using averaging procedure and the other smoothed using morphological opening filter. The spectrum $P_{0}(t, f)$ changing with time is obtained by Short-Time Fourier Transform (STFT) of the original signal $f(t) . P_{0}(t, f)$ is converted to the auto-spectrum $\left|P_{0}(t, f)\right|$ using the conjugate operation. Using the old method ${ }^{1)}$, averaging procedure has been employed to smooth the auto-spectrum as shown in (a). The value $w$ in this figure is the time-width in the averaging procedure; it is also called the analyzing width in this paper. On the other hand, (b) shows the proposed method for obtaining the lofargram output $|P(t, f)|$ using morphological opening filter by the structuring element $g(t, f)$. The morphological opening filter of the auto-spectrum $\left|P_{0}(t, f)\right|$ by the structuring element $g(t, f)$ is defined as follows ${ }^{11)}$,

$$
O\left(\left|P_{0}(t, f)\right|, g(t, f)\right)=\left|P_{0}(t, f)\right| \ominus \hat{g}(t, f) \oplus g(t, f)
$$

$$
\left|P_{0}(t, f)\right| \oplus g(t, f)=\sup _{(\tau, \phi \in B)}\left\{\left|P_{0}(t-\tau, f-\phi)\right|+g(\tau, \phi)\right\}
$$

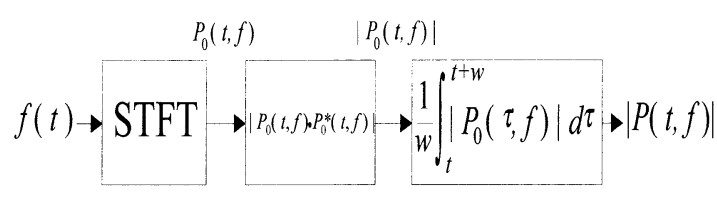

STFT : Short-Time Fourier Transform

(a) with averaging procedure

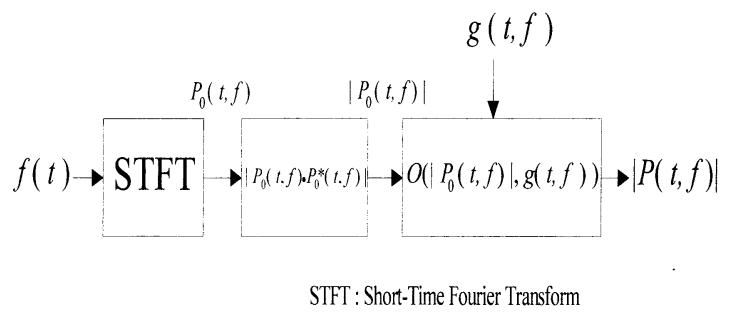

(b) with morphlogical opening filter

Fig. 1 Block diagrams of lofargram. 


$$
\left|P_{0}(t, f)\right| \ominus g(t, f)=\inf _{(\tau, \phi) \in B}\left\{\left|P_{0}(t-\tau, f-\phi)\right|-g(\tau, \phi)\right\}
$$

where $B$ is the support of the structuring element $g(t, f)$. The effect of the smoothing is determined by the structuring elements in opening filter, and the functions were used in the past studies as follows;

$$
g_{1}(t, f)=\left\{\begin{array}{cc}
0 & \left(-\frac{w}{2} \leq t \leq \frac{w}{2}, f=0\right) \\
-\infty & \left(t<-\frac{w}{2}, \frac{w}{2}<t, f=0\right) \\
-\infty & (f \neq 0)
\end{array}\right.
$$

$$
g_{2}(t, f)=\left\{\begin{array}{l}
0\left(t-f=0,-\frac{w}{2 \sqrt{2}} \leq t \leq \frac{w}{2 \sqrt{2}},-\frac{w}{2 \sqrt{2}} \leq f \leq \frac{w}{2 \sqrt{2}}\right) \\
-\infty\left(t-f=0, t<-\frac{w}{2 \sqrt{2}}, \frac{w}{2 \sqrt{2}}<t, f<-\frac{w}{2 \sqrt{2}}, \frac{w}{2 \sqrt{2}}<f\right) \\
-\infty \quad(t-f \neq 0) .
\end{array}\right.
$$

Since the values of these functions show only zero, we can redefine the morphological opening filter using the support $B$ of the structuring element $g(t, f)$. The morphological opening filter of the auto-spectrum $\left|P_{0}(t, f)\right|$ by the structuring element $B$ is defined as follows;

$$
\begin{aligned}
& O\left(\left|P_{0}(t, f)\right|, B\right)=\left|P_{0}(t, f)\right| \ominus \hat{B} \oplus B \\
& \left|P_{0}(t, f)\right| \oplus B=\sup _{(\tau, \phi) \in B}\left\{\left|P_{0}(t-\tau, f-\phi)\right|\right\} \\
& \left|P_{0}(t, f)\right| \ominus B=\inf _{(\tau, \phi) \in B}\left\{\left|P_{0}(t-\tau, f-\phi)\right|\right\}
\end{aligned}
$$

where the structuring element $B$ is a subspace of time-frequency space, which is the domain of the lofargram. Thus, eqs. (4) and (5) are also redefined as follows;

$$
\begin{aligned}
& B_{1}=\left\{(t, f) \mid f=0,-\frac{w}{2} \leq t \leq \frac{w}{2}\right\} \\
& B_{2}=\left\{(t, f) \mid t-f=0,-\frac{w}{2 \sqrt{2}} \leq t \leq \frac{w}{2 \sqrt{2}},-\frac{w}{2 \sqrt{2}} \leq f \leq \frac{w}{2 \sqrt{2}}\right\}
\end{aligned}
$$

These are line segments in which both measures are $w$ and each slope is different. The measure $w$ is also called the analyzing width, as it was in the case of the averaging procedure. Wang et al. have derived the output probability distribution function of multilevel morphological opening filter when the structuring element is a line segment ${ }^{8)}$. The probability density function of the auto-spectrum is transformed into that of the lofargram output using this property. It can be possible to derive the theoretical equations of the average, the variance, and the processing gain. If the measure of the discrete structuring element is $w$ and the probability distribution function of the auto-spectrum $\left|P_{0}(t, f)\right|$ corresponding to the level $\ell$ is described as $F_{0}(\ell)$, which is the same function on any $(t, f)$, the probability distribution function $F_{\text {ouput }}(\ell)$ of the lofargram output $|P(t, f)|$ is expressed as follows ${ }^{8)}$,

$$
F_{\text {output }}(\ell)=1-w\left[1-F_{0}(\ell)\right]^{w}+(w-1)\left[1-F_{0}(\ell)\right]^{w+1} .
$$

Here, the level $\ell$ is the value taken by the auto-spectrum $\left|P_{0}(t, f)\right|$, and it is a positive real number.

Therefore, we can derive the processing gain of the lofargram output using the probability density function of the auto-spectrum. First, the probability distribution function $F_{0}(\ell)$ is obtained by the probability density function $p_{0}(\ell)$ of the auto-spectrum as follows;

$$
F_{0}(\ell)=\int_{-\infty}^{\ell} p_{0}(x) d x
$$

By applying the above equation to eq. (11), 
the probability distribution function $F_{\text {oupu }}(\ell)$ is obtained. The probability density function $p_{\text {ouput }}(\ell)$ of the lofargram output is then obtained by differentiating $F_{\text {output }}(\ell)$ with respect to $\ell$.

$$
p_{\text {output }}(\ell)=\frac{d}{d \ell} F_{\text {output }}(\ell)
$$

Let the level of the lofargram output be the random variable $n(w)$. The probability distribution function $F_{0}(\ell)$ is the probability that $n(w) \leq \ell$ will happen at level $\ell$. The average $\bar{n}(w)$ of the lofargram output corresponding to the analyzing width (the measure of the structuring element) $w$ is

$$
\bar{n}(w)=E[n(w)]=\left\{\begin{array}{cc}
\int_{-\infty}^{\infty} \ell p_{0}(\ell) d \ell & (w=1) \\
\int_{-\infty}^{\infty} \ell p_{\text {output }}(\ell) d \ell & (w \geq 2)
\end{array}\right.
$$

And the variance $\sigma_{n}^{2}(w)$ of the lofargram output is

$$
\begin{aligned}
\sigma_{n}^{2}(w) & =E\left[(n(w)-E[n(w)])^{2}\right] \\
& =\left\{\begin{array}{cc}
\int_{-\infty}^{\infty}(\ell-\bar{n}(1))^{2} p_{0}(\ell) d \ell & (w=1) \\
\int_{-\infty}^{\infty}(\ell-\bar{n}(w))^{2} p_{\text {output }}(\ell) d \ell & (w \geq 2)
\end{array}\right.
\end{aligned}
$$

Where, $E[]$ is the expected value. The processing gain corresponding to the analyzing width $w$ is defined by the difference of the minimum detection level between the analyzing width 1 and $w^{9)}$.

$$
P G(w)=10 \log _{10} \frac{\bar{n}(1)}{\bar{n}(w)}+10 \log _{10} \frac{\sigma_{n}(1)}{\sigma_{n}(w)}
$$

Here, $\bar{n}(1)$ and $\sigma_{n}(1)$ are the average and the standard deviation without smoothing, respectively.

\section{Comparison of the computer simu- lation with the theoretical value}

\subsection{The theoretical equation of processing gain with Gaussian noise input}

It is generally known that the ambient noise in an ocean is stationary and Gaussian in short time ${ }^{12)}$, ${ }^{13)}$. Here, we assume that the input signal is Gaussian noise. The probability distribution of the lofargram output is then obtained, and the theoretical equation is derived. The spectrum fluctuating with time is obtained by STFT in the lofargram. In general, the input signal cut out by the window function shifts with time, and the power spectrum of the signal is obtained using FFT. Since Fourier transform is a linear transform, when the input signal $f(t)$ is Gaussian noise, both the real and imaginary parts of its Fourier spectrum are random variables that obey Gaussian distribution such that the averages are zero and the variances are the same value. Let the real and imaginary parts of the spectrum $P_{0}(t, f)$ be described as $\operatorname{Re}\left[P_{0}(t, f)\right]$ and $\operatorname{Im}\left[P_{0}(t, f)\right]$, respectively. The power spectrum (auto-spectrum) is expressed by $\left|P_{0}(t, f)\right|=\operatorname{Re}\left[P_{0}(t, f)\right]^{2}+\operatorname{Im}\left[P_{0}(t, f)\right]^{2}$. It is known that the square sum of the independent Gaussian random variable obeys $\chi^{2}$ distribution. If both the real and imaginary parts of $P_{0}(t, f)$ are the independent Gaussian random variables such that the average is zero and the variance is $v^{2}$

$\left\{\operatorname{Re}\left[P_{0}(t, f)\right]^{2}+\operatorname{Im}\left[P_{0}(t, f)\right]^{2}\right\} / v^{2}$ obeys $\chi^{2}$ distribution with two degree of freedom. The probability density function $p_{0}(\ell)$ of the auto-spectrum $\left|P_{0}(t, f)\right|$ is

$$
p_{0}(\ell)=\frac{1}{2 v^{2} \Gamma(1)} \exp \left(-\frac{\ell}{2 v^{2}}\right)
$$

By applying the above equation to eqs. (11)(16), the theoretical equation of the processing gain can be derived. By integrating eq. (17) such as in eq. (12), the probability distribution function $F_{0}(\ell)$ of the auto-spectrum $\left|P_{0}(t, f)\right|$ is obtained as follows; 


$$
F_{0}(\ell)=1-\exp \left(-\frac{\ell}{2 v^{2}}\right)
$$

The probability distribution function $F_{\text {output }}(\ell)$ of the lofargram output is obtained by applying eq.

(18) to eq. (11).

$$
F_{\text {output }}(\ell)=1-w \exp \left(-\frac{w}{2 v^{2}} \ell\right)+(w-1) \exp \left(-\frac{w+1}{2 v^{2}} \ell\right)
$$

The probability density function $p_{\text {output }}(\ell)$ of the lofargram output is obtained by differentiating eq. (19) such as in eq. (13).

$$
p_{\text {output }}(\ell)=\frac{w^{2}}{2 v^{2}} \exp \left(-\frac{w}{2 v^{2}} \ell\right)-\frac{w^{2}-1}{2 v^{2}} \exp \left(-\frac{w+1}{2 v^{2}} \ell\right)
$$

The average $\bar{n}(w)$ corresponding to the analyzing width $w$ is obtained by applying eq. (20) to eq. (14).

$$
\bar{n}(w)=\frac{4 v^{2}}{w+1}
$$

By applying eqs. (20) and (21) to (15), the variance $\sigma_{n}^{2}(w)$ corresponding to the analyzing width $w$ is obtained.

$$
\sigma_{n}^{2}(w)=\frac{8 v^{4}}{w(w+1)}
$$

Finally, the processing gain is derived by applying eqs. (21) and (22) to (16) as follows;

$$
P G(w)=10 \log _{10} \frac{w+1}{2}+10 \log _{10} \sqrt{\frac{w(w+1)}{2}}
$$

\subsection{The computer simulation and theo- retical value}

In the computer simulation, the auto-spectrum is obtained by FFT of Gaussian noise generated on the computer. The smoothing performed by the morphological opening filter is calculated with the structuring element of eq. (9) over the time-axis on the lofargram. Table 1 shows the average and the standard deviation, including the processing gain in the computer simulation. In the same manner as in past studies $10), 11)$, both the average and the standard deviation of the lofargram decrease as the analyzing width increases. This confirms that the morphological opening filter increases the processing gain in the computer simulation results. Table 1 also lists the theoretical values of the average, the standard deviation, and the processing gain obtained by eqs. (21)-(23). These results are approximately similar to those of the computer simulation. The relative error of the processing gain between the computer simulation and the theoretical value is also shown in Table 1 . The relative error is obtained such that the absolute of the difference between the simulation results and the theoretical value is divisible by the theoretical value. Since in these results the maximum relative error is approximately $1.083[\%]$, the computer simulation results agree closely with the theoretical values. Therefore,

Table 1 Average, standard deviation, and processing gain.

\begin{tabular}{|c|c|r|r|r|r|r|}
\hline \multicolumn{2}{|c|}{ Analyzing Width $w$} & \multicolumn{1}{c|}{1} & \multicolumn{1}{c|}{3} & \multicolumn{1}{c|}{5} & \multicolumn{1}{c|}{9} & \multicolumn{1}{c|}{15} \\
\hline \multirow{4}{*}{ Simulation } & Average $\bar{n}(w) \times 10^{-4}$ & 313.337 & 155.099 & 103.959 & 61.963 & 38.750 \\
\cline { 2 - 7 } & Standard Deviation $\sigma_{n}(w) \times 10^{-4}$ & 313.311 & 126.998 & 81.129 & 45.731 & 28.131 \\
\cline { 2 - 7 } & Processing Gain $P G(w)[\mathrm{dB}]$ & 0.000 & 6.975 & 10.659 & 15.396 & 19.545 \\
\hline \multirow{3}{*}{$\begin{array}{c}\text { Theretical } \\
\text { Value }\end{array}$} & Average $\bar{n}(w) \times 10^{-4}$ & 313.335 & 156.667 & 104.445 & 62.667 & 39.166 \\
\cline { 2 - 7 } & Standard Deviation $\sigma_{n}(w) \times 10^{-4}$ & 313.335 & 127.918 & 80.902 & 46.709 & 28.603 \\
\cline { 2 - 7 } & Processing Gain $P G(w)[\mathrm{dB}]$ & 0.000 & 6.901 & 10.651 & 15.255 & 19.426 \\
\hline \multicolumn{2}{|c|}{ Relative Error [\%] } & 0.000 & 1.083 & 0.073 & 0.921 & 0.609 \\
\hline
\end{tabular}


we can also theoretically confirm that the processing gain is improved by use of the morphological opening filter.

Since in the computer simulation the variance $v^{2}$ of both the real and imaginary parts of the auto-spectrum are approximately 156.667 $\times 10^{-4}$, the probability density function of the lofargram output is obtained by substituting it into eq. (20). Fig. 2 shows the probability density function and histogram obtained by the computer simulation. As the analyzing width increases, the peak of the distribution shifts to the left side and the width of the distribution is reduced. This shows that both the average and the variance decrease as the analyzing width increases using the morphological opening filter. The goodness-of-fit test is then performed based on the hypothesis that the theoretical probability density function agrees with the histogram obtained by the computer simulation. The hypothesis is accepted at a level of significance of 5 [\%] under all conditions. Therefore, it is clear that the theoretical probability density function agrees closely with the histogram obtained by the computer simulation. Furthermore, the probability density function and histogram of the lofargram output using the averaging procedure are shown in Fig 3. Since the random variable of $\chi^{2}$ distribution with two degree of freedom such as was seen in eq. (17), is averaged over $w$ times, its probability density function becomes the $\chi^{2}$ distribution with $2 w$ degree of freedom as follows;

$$
p_{\text {averaging }}(\ell)=\frac{2}{2^{w} v^{2} \Gamma(w)}\left(\frac{w}{v^{2}} \ell\right)^{w-1} \exp \left(-\frac{w}{2 v^{2}} \ell\right)
$$

The average is $4 v^{2}$, which is the same as any $w$. And the variance is $4 v^{2} / w$. Therefore, the processing gain using the averaging procedure is

$$
P G_{\text {averaging }}(w)=10 \log _{10} \sqrt{w}
$$

This shows that the processing gain using the averaging procedure is improved by only decreas- ing the variance of the lofargram output. In Fig. 3, the noise components approach the average of $313.337 \times 10^{-4}$ as the analyzing width $w$ increases. The peak of the distribution does not reach zero, as seen in Fig. 2. Examples of the lofargram output are shown in Fig. 4. The target signal is the sine wave added onto the same Gaussian noise as that seen in Figs. 2 and 3. The $\mathrm{S} /$ $\mathrm{N}$ ratio is $-30[\mathrm{~dB}]$. Here, the source signal is $f(t)=a(t)+b(t)$, the target signal is $a(t)$, the noise is $b(t)$, and the $\mathrm{S} / \mathrm{N}$ ratio is

$20 \log _{10}\left(E\left[a(t)^{2}\right] / E\left[b(t)^{2}\right]\right)$. In the averaging results, the larger the averaging width, the wider the noise components were spread on the time-axis. We can also confirm that the noise components near the average of $313.337 \times 10^{-4}$ as seen in Fig. 4. It is difficult to distinguish the target signal from the lofargram outputs. Thus it can be considered that, when the averaging procedure is used, there is no effect on the noise suppression that corresponds to the large analyzing width $w$. On the other hand, the sine wave component of the target signal can be detected in the results regarding the morphological opening filter. As the morphological opening filter increases the difference between the target signal level and the noise component levels, the visualization effect of the lofargram is improved. Therefore, it is useful to lessen the threshold detection level to determine the weak target. It follows from what has been said that the morphological opening filter is more suitable than the averaging procedure for the smoothing of the lofargram.

\section{Conclusions}

As stated above, the following results were obtained.

- The theoretical equation regarding the processing gain was derived when the source signal was Gaussian noise in the smoothing process using the morphological opening filter.

- It was clarified theoretically that both the average and the variance (standard deviation) of the noise components decrease as the analyzing width increases. 


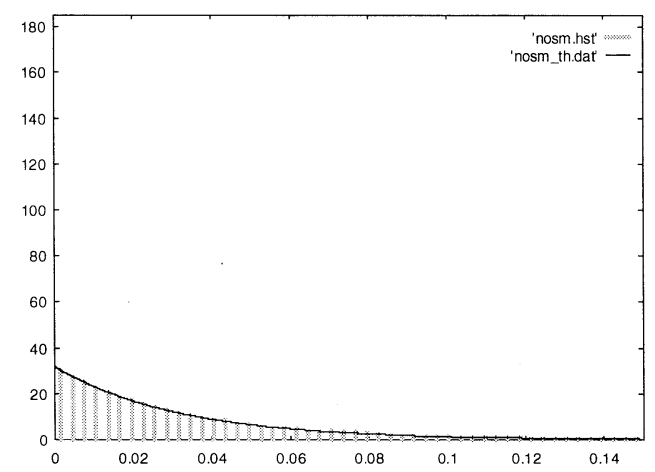

(a) no smoothing

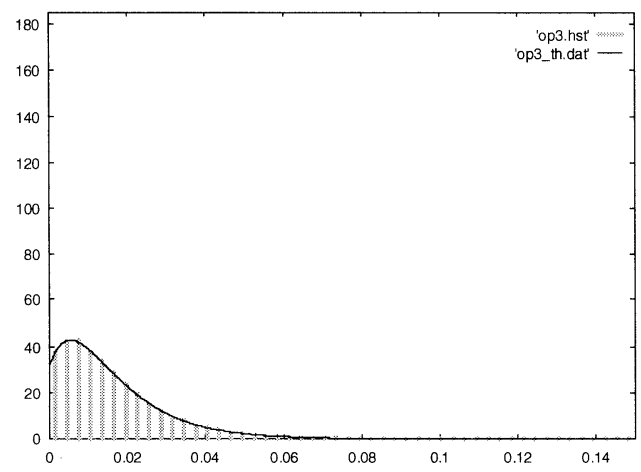

(b) $w=3$

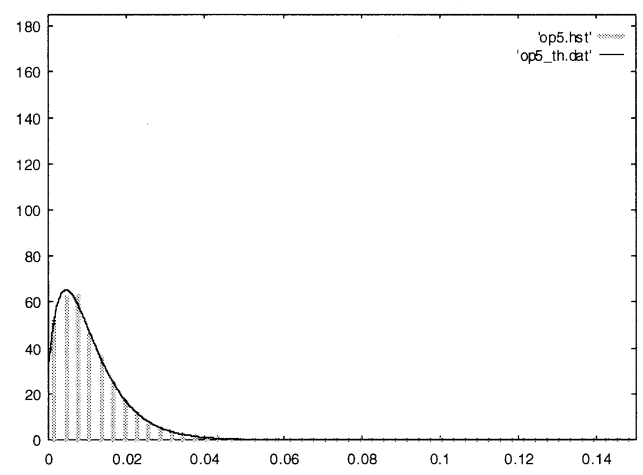

(c) $w=5$

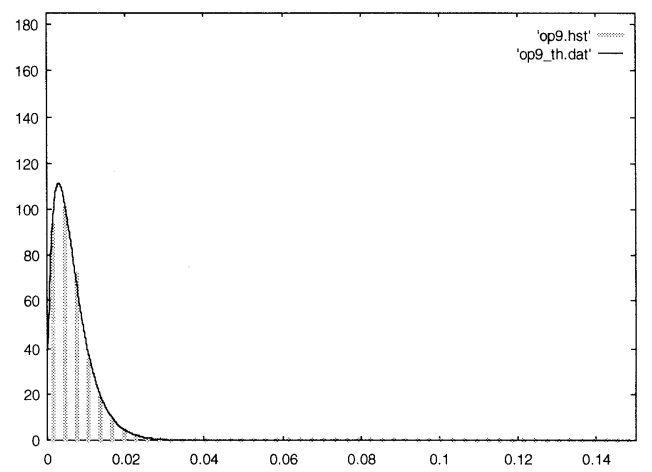

(d) $w=9$

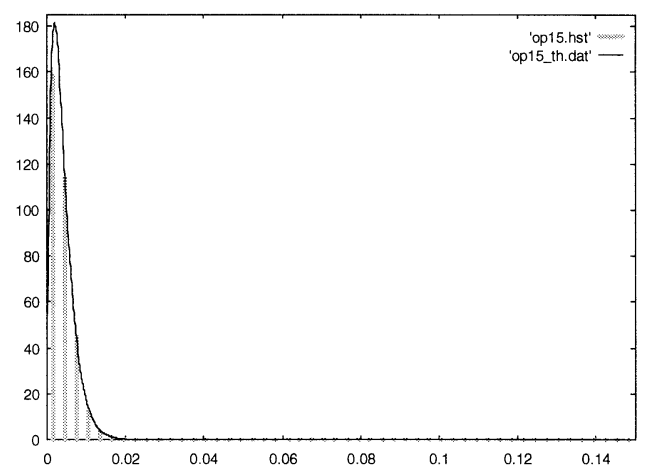

(e) $w=9$

Fig. 2 Comparison between theoretical probability density function and histogram of simulation using morphological opening filter (The horizontal axis is the level of lofargram output. The vertical axis is the number of times at each level.). 


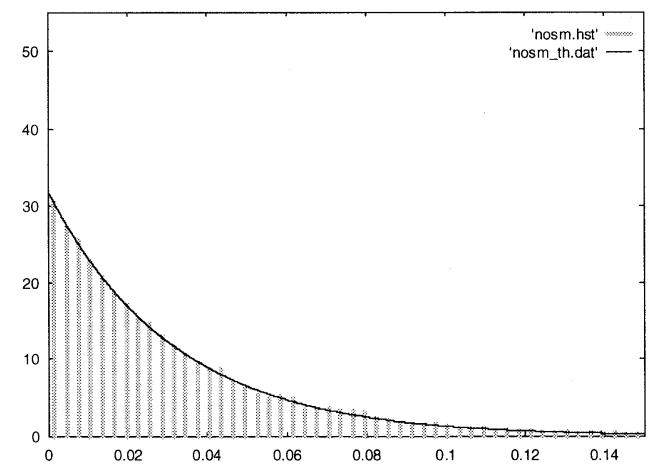

(a) no smoothing

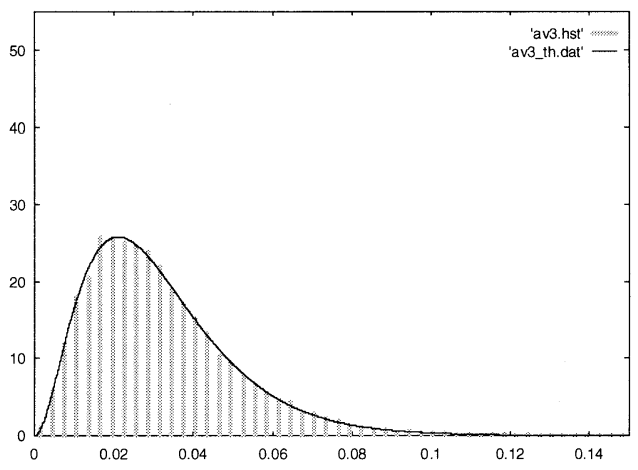

(b) $w=3$

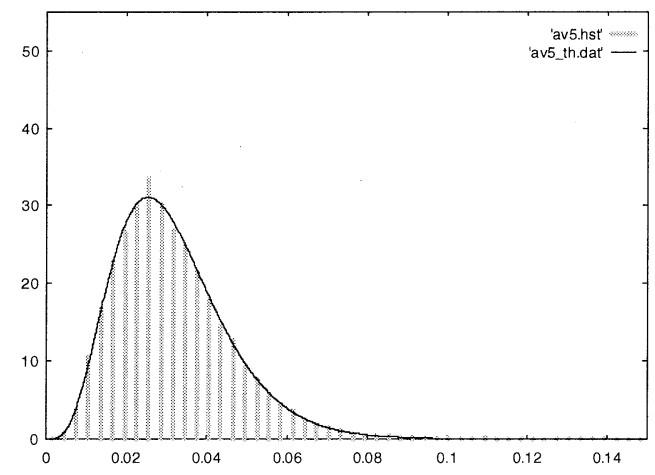

(c) $w=5$

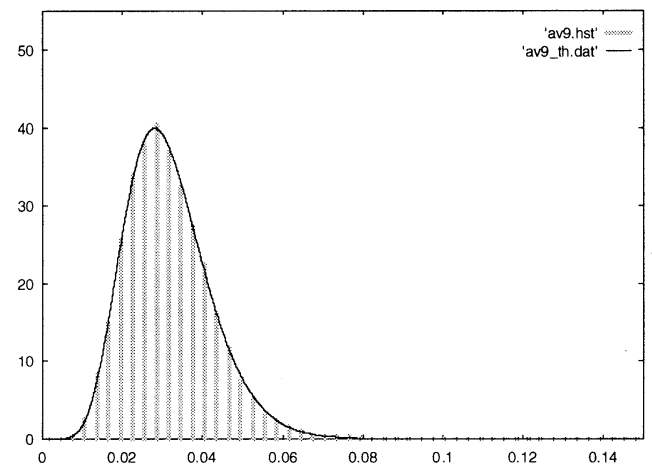

(d) $w=9$

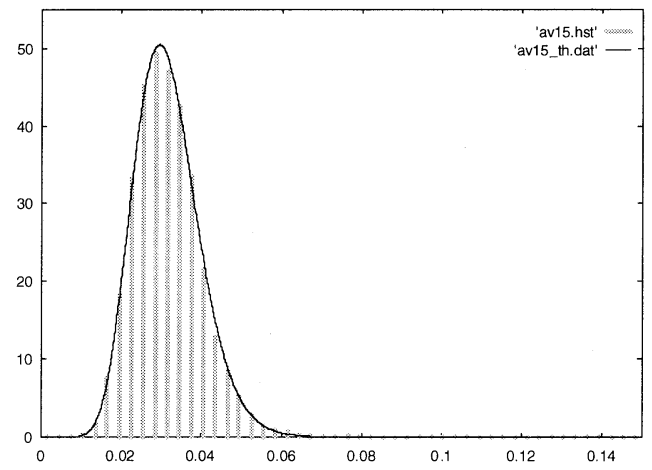

(e) $w=15$

Fig. 3 Comparison between theoretical probability density function and histogram of simulation using averaging procedure (The horizontal axis is the level of lofargram output. The vertical axis is the number of times at each level.). 

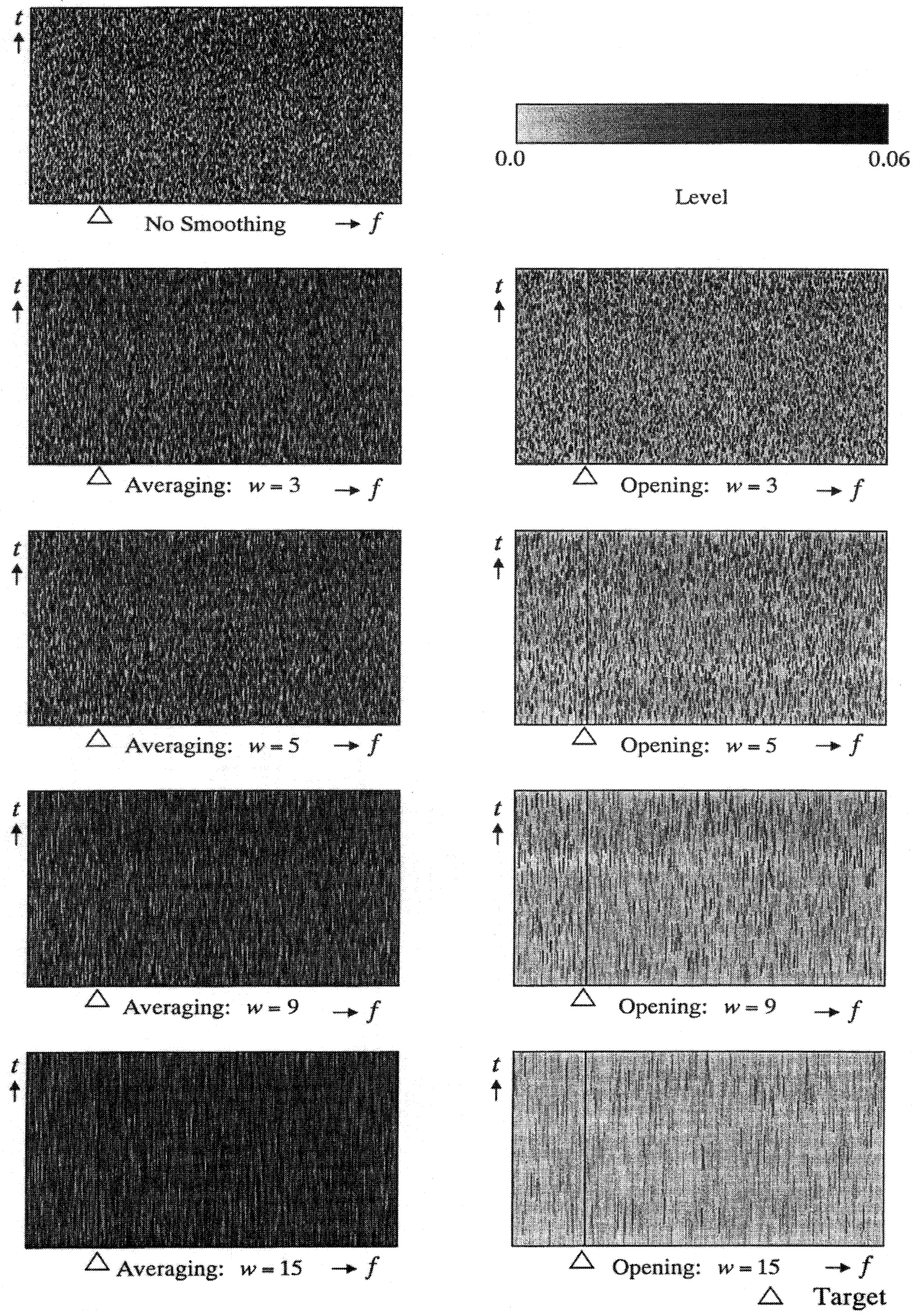

Fig. 4 Examples of lofargram output. 
- Since the maximum relative error of the processing gain was approximately 1.083 [\%], the computer simulation results agreed closely with the theoretical values.

Therefore, we can consider that the theoretical equation to obtain the processing gain derived in this paper is accurate. It was quite time consuming to analyze the noise suppression effect of the lofargram smoothing method using the morphological opening filter by means of computer simulation. The results of this study show that processing gain can be evaluated quickly and easily. Thus, it is useful to clarify the noise suppression effect of the lofargram smoothing method using the morphological opening filter by analyzing the noises at any other probability distribution in the same way.

\section{Reference}

1) Alan V. Oppenheim Editor, Applications of Digital Signal Processing (Prentice-Hall, 1978), pp. 406-416.

2) The Marine Acoustics Society of Japan editor, MARINE ACOUSTICS - Fundamentals and Applications -, (The Marine Acoustics Society of Japan, 1984), pp. 99 (in Japanese).

3) Richard O. Nielsen, SONAR SIGNAL PROCESSING (Artech House, 1991), pp. 158-173.

4) J. Serra, "Image Analysis and Mathematical Morphology", (Academic Press, 1982).

5) Petros Maragos, "A Representation Theory for Morphological Image and Signal Processing",IEEE TRANSACTIONS ON PATTERN ANALYSIS AND MACHINE INTELLIGENCE, 11(6), 586-599 (1989).

6) Rovert L. Stevenson and Gonzalo R. Arce, "Morphological Filters: Statistics and Further Syntactic Properties" , IEEE TRANSACTION ON CIRCUITS AND SYSTEMS, VOL. CAS-34(11), 12921305,(1987).
7) Olli Yli-Harja, Jaakko Astola and Yrjo Neuvo, "Analysis of the Properties of Median and Weighted Median Filters Using Threshold Logic and Stack Filter Representation" , IEEE TRANSACTIONS ON SIGNAL PROCESSING, 39( 2), 395-410 (1991).

8) Demin Wang, Veronique Haese-Coat, Alain Bruno, and Joseph Ronsin, "Some Statistical Properties of Mathematical Morphology", IEEE TRANSACTIONS ON SIGNAL PROCESSING, 43(8), 19551965 (1995).

9) Yoshikazu Kohama, Mamoru Egawa, Yuuichi Shiraki, "Theoretical study of processing gain by averaging in the frequency analysis" ,The Journal of The Marine Acoustics Society of Japan, 21( 1), 35-43 (1994) (in Japanese).

10) Kazuyoshi Mori, Minoru Matsuda, “A Smoothing Method of Lofargram using Morphological Opening Filter" , The Journal of The Marine Acoustics Society of Japan, 23(2), 94-107 (1996) (in Japanese).

11) Kazuyoshi Mori, Minoru Matsuda, "A Smoothing Method of Lofargram using Morphological Opening Filter" , (Part 2) Utilization of Structuring Element on Time-Frequency Space", The Journal of The Marine Acoustics Society of Japan, 23(4), 220-229 (1996) (in Japanese).

12) Robert J. Urick, Principles of Underwater Sound, (McGraw-Hill, Inc., 1967).

13) The Marine Acoustics Society of Japan editor, MARINE ACOUSTICS - Fundamentals and Applications -, (The Marine Acoustics Society of Japan, 1984), pp. 99 (in Japanese). 